\title{
Detection of HER-2/neu Amplification on Fine Needle Aspirates of Breast Cancer Using Fluorescence in Situ Hybridization ${ }^{*}$
}

\author{
Talaat Iman $^{1 \#}$, Sorour Amani ${ }^{2}$, Abdel-Hadi Mona ${ }^{1}$ \\ ${ }^{1}$ Departments of Pathology, Faculty of Medicine, Alexandria University, Alexandria, Egypt; ${ }^{2}$ Clinical Pathology, Alexandria Univer- \\ sity, Alexandria, Egypt. \\ Email: "Iman_talaat@yahoo.com
}

Received June $3^{\text {rd }}, 2013$; revised July $5^{\text {th }}, 2013$; accepted July $12^{\text {th }}, 2013$

Copyright (C) 2013 Talaat Iman et al. This is an open access article distributed under the Creative Commons Attribution License, which permits unrestricted use, distribution, and reproduction in any medium, provided the original work is properly cited.

\begin{abstract}
The accuracy of diagnostic assays for HER-2/neu in breast cancer is extremely important as HER-2/neu status is essential in tailoring adjuvant and/or neoadjuvant treatment in every patient. FNAC is widely practiced in Egypt in preoperative diagnosis of breast cancer for its low cost and high diagnostic accuracy. Since the determination of HER-2/neu protein expression on cytological preparations was previously found to be unreliable for clinical use, we opted for the assessment of HER-2/neu status in fine needle aspirates using FISH. The main objective of this study was to evaluate the reliability of HER-2/neu status assessment by FISH on fine needle aspirates of breast cancers by comparing the results with IHC and FISH on FFPE tissue sections obtained from corresponding surgically resected specimens. Fine needle aspirates from 40 breast cancer patients with pathologically confirmed breast cancer were included in the study. They were submitted for HER-2/neu evaluation by FISH. After surgery, the corresponding FFPE sections were evaluated for HER-2/neu by FISH and by IHC. FNAs from 11 cases proved to be amplified by FISH, while 29 cases were not amplified. Apart from two cases that showed lack of signals, all specimens evaluated by FISH on the corresponding FFPE sections showed matched results. The Measurement of Agreement between FISH on FNAs and FISH on FFPE sections was $86.7 \%$, while that between FISH on FNAs and IHC was $72.5 \%$. The high concordance rate in the present study between FISH evaluation of HER-2/neu gene amplification on FNAC samples and their corresponding FFPE samples indicate that FISH may be a reliable technique for HER-2/neu assessment on FNAs. Furthermore, FISH on FNAs gave us better hybridization signals than their corresponding FFPE tissue sections. Finally, we also conclude that all score $(2+)$ cases by IHC should be reevaluated by FISH which is crucial for the patient management.
\end{abstract}

Keywords: Breast Cancer; FISH on FNA; Immunohistochemistry; HER-2/neu

\section{Introduction}

Breast cancer is one of the major health problems in Egypt and is by far the most common type of cancer in Egyptian women [1,2]. The development of breast cancer is associated with a number of genetic alterations involving the inactivation of tumour suppressor genes and the activation of oncogenes. A dominant mechanism, leading to oncogene activation is the amplification of specific genomic region [3].

The ERBB2 (HER-2/neu) oncogene is amplified and overexpressed in about $25 \%$ of invasive breast carcino-

\footnotetext{
"No conflict of interests. No external funds were received, and all expenses were paid by the researchers.

${ }^{\#}$ Corresponding author.
}

mas. In general, ERBB2 amplification confers unfavorable prognosis, although its significance is less than that of the traditional prognostic factors - stage and grade [4]. There are two main methods for establishing HER-2/neu status in the diagnostic setting. HER-2/neu protein expression can be detected using immunohistochemistry (IHC), and HER-2/neu gene amplification can be quantified using in-situ hybridization (ISH) with either fluorescence (FISH) or chromogenic (CISH) probe detection [5]. The relationship between HER-2/neu protein levels and response to treatment is proportional to the degree of over expression [6,7]. Protein expression level correlates with HER-2/neu gene copy number, and IHC has been shown to be reliable in detecting increased protein expression in 
cases with a high level of gene amplification [8,9]. However scoring of HER-2/neu IHC is highly subjective and inter-observer reproducibility can be problematic, especially for $2+$ cases [10].

FISH is a molecular technique performed on formalin-fixed paraffin-embedded (FFPE) tissues, frozen sections as well as fine needle aspirate biopsy (FNAB), which permits the direct visual assessment of gene copy number in interphase and metaphase nuclei [11]. DNA is more stable than protein, making FISH less fixation sensitive than IHC [12]. Gene amplification correlates directly with protein and mRNA levels, and FISH has been shown to be superior to IHC in predicting clinical outcome and response to trastuzumab therapy [13]. However, FISH has some disadvantages, one of them that it is much more expensive than IHC, although given the high cost of trastuzumab therapy, cost-effectiveness analysis has suggested that FISH testing all $2+/ 3+$ cases and avoiding unnecessary treatment of false-positive cases may save money [14].

Preoperative determination of the HER-2/neu status is becoming increasingly important to plan for the type of surgery as well as for neoadjuvant chemotherapy in selected patients. Preoperative HER-2/neu testing can be done by IHC on FFPE core needle biopsies (CNB). Although the test was found to be successful on CNB [15], still some obstacles exist: The ASCO/CAP guidelines recommend that cores entirely involved by retraction artifacts or crush artifacts should not be used as a sample to perform/interpret HER-2/neu immunohistochemistry [16] and the UK recommendations precise that observers should be aware of the range of common artifacts, including edge artifacts, which can be problematic in small biopsy samples [17].

In Egypt, breast FNAB plays a major role in the diagnosis of both palpable and non-palpable breast masses because cost-efficient health care is a priority in this country and the less expensive but effective diagnostic methods are preferred. Furthermore Breast FNAB has proved to have a very goodspecificity, sensitivity, and diagnostic accuracy [18]. And therefore in our situation it would be useful to be able to identify HER-2/neu status by using FNAB specimens.

Unfortunately, HER-2/neu protein expression on cytological preparations with any of the fixatives was found to be insufficiently reliable for clinical use [18]. On the other hand, several studies have reported successful use of FISH on FNA cytological material to evaluate HER2/neu amplification [19-23]. Yet, few studies compared the efficacy of FISH on FNA specimens and on corresponding FFPE tissue samples [18].

\section{Objectives}

The objective of this study was to evaluate the reliability of HER-2/neu status assessment by FISH on FNAs of breast cancers by comparing the results with FISH and IHC done on FFPE tissue sections obtained from corresponding surgically resected specimens. We also aimed at evaluating concordance rates between IHC HER-2/neu positive and IHC HER-2/neu negative cases with FISH amplified and non-amplified cases respectively, in order to validate the HercepTest ${ }^{\mathrm{TM}}$ that is newly implemented in our laboratory.

\section{Material and Methods}

Between November, 2011 and May, 2012, fine needle aspirates were collected from 43 female patients having an American College of Radiology Breast Imaging Reporting and Data System (BIRADS) score of 5 on mammogram and undergoing preoperative confirmation of malignancy. All patients signed informed consents. Two FNA samples were subsequently discarded from the study because they were hypocellular, and one sample was also excluded because the post-operative biopsy showed an in situ ductal carcinoma.

Fine needle aspirates from the remaining 40 breast cancer patients were included in the study. They were submitted for routine diagnostic cytology as well as for HER-2/neu evaluation by FISH. After surgery, the corresponding sections of archival formalin-fixed, paraffinembedded tissue were evaluated for HER-2/neu by FISH and by IHC.

\subsection{Sample Preparation}

1) FNA sample collection: Preoperative samples were collected by FNA under ultrasound guidance using a 23 -gauge needle. Ultrasound guidance was used in all cases to ascertain the aspiration of sufficient amount of cells from within the tumor itself and not from any surrounding breast tissue. The aspirated material was smeared on glass slides, two of which positively charged. The 2 smears on positively charged slides were fixed in methanol/glacial acetic acid (3:1) and stored in $-20^{\circ} \mathrm{C}$ until their use for FISH. The remaining slides were fixed in alcohol for routine $\mathrm{H} \& \mathrm{E}$ staining.

2) FFPE tissue sections: Postoperative tissue biopsies were fixed in $10 \%$ buffered formalin overnight and then were processed for paraffin embedding. Tissue blocks were then cut into $4 \mu \mathrm{m}$ thick sections on positively charged slides, and incubated overnight at $56^{\circ} \mathrm{C}$.

\subsection{HER2/neu Immunohistochemistry}

Immunohistochemical analysis was performed manually on FFPE tissue sections using the FDA-approved HercepTest $^{\mathrm{TM}}$, according to the manufacturer's instructions.

Briefly, following deparaffinization and rehydration, 
the tissue sections were subjected to heat-activated epitope retrieval in citrate buffer at $96^{\circ} \mathrm{C}$ for 30 minutes. Blocking of endogenous peroxidase preceded the addition of the primary anti-HER-2/neu antibody (rabbit antihuman c-erbB-2 A0485, Dako, Copenhagen, Denmark) at $1 / 100$ dilution. Binding of the primary antibody was revealed by means of the chromogen substrate, 3,3'-diaminobenzidine (DAB). Slides were then counterstained with hematoxylin. A positive tissue control section was included in each run of immunohistochemical staining, as well as a negative control slide in which the primary antibody was omitted.

\subsection{Scoring of Immunohistochemical Results}

The slides were scored independently by 2 histopathologists (the first and the third authors) according to the ASCO \& CAP guidelines [15] as follows.

\subsubsection{Score 3 (Positive)}

Uniform intense membrane staining of more than $30 \%$ of invasive tumor cells. A homogeneous (chicken wire) pattern should be present.

\subsubsection{Score 2 (Equivocal)}

Complete membrane staining that is non-uniform or weak but with obvious circumferential distribution in at least $10 \%$ of cells, or intense complete membrane staining in $<30 \%$ of tumor cells.

\subsubsection{Score 1 (Negative)}

Weak, incomplete membrane staining in any proportion of invasive tumor cells, or weak, complete membrane staining in less than $10 \%$ of cells.

\subsubsection{Score 0 (Negative)}

No staining is observed in invasive tumor cells.

Before we started testing the patients included in the study we retrospectively tested 30 patients with known HER2/neu FISH status using the FDA-approved DakoHercepTest ${ }^{\mathrm{TM}}$ for immunohistochemistry. Some technical issues were slightly modified as well as the interpretation criteria in order to meet the ASCO/CAP guidelines recommendations. A concordance rate of $90 \%$ was reached. The $10 \%$ of cases that showed lack of agreement ( 3 cases) were in the category of $2+$ by IHC. All assay procedures were standardized before starting the new cases.

\subsection{FISH Protocol}

1) For cytological specimens: Slides were brought to room temperature and incubated in $2 \mathrm{xSSC}$ at $37^{\circ} \mathrm{C}$ for 30 min, gradually dehydrated in alcohol, air dried and proc- essed by FISH.

2) For FFPE tissue sections: deparaffinization, pretreatment, enzyme digestion and fixation of slides were performed using the Vysis Paraffin Pretreatment Kit (Vysis) according to the manufacturer's recommended protocol.

\subsection{Probe Application and Hybridization}

Denaturation and hybridization were carried out in a HYBrite Denaturation/Hybridization System for FISH (Vysis). All slides were denaturated at $72^{\circ} \mathrm{C}$ for $2 \mathrm{~min}$. HER-2/neu probe mix (10 $\mu$ l for FFPE sections and $3 \mu \mathrm{l}$ for FNAC slides) was added and hybridization took place at $37^{\circ} \mathrm{C}$ for $14-18 \mathrm{~h}$. The slides were then washed in post-hybridization wash buffer at $72^{\circ} \mathrm{C}$ for $2 \mathrm{~min}$ and counterstained with DAPI/antifade.

\subsection{Evaluation of the FISH Signals}

In each case, 100 - 200 well preserved, separately located interphase cells with clearly visible distinct signals were scored for HER-2/neu and chromosome 17 signals. FISH signals were analyzed using BX51/61 Olympus fluorescent microscope equipped with a suitable set of filters including: DAPI single band pass, dual band pass FITC/ TRIC and triple band pass (FITC/TR/DAPI) (Olympus, UK LTD).

Only single, non-overlapping and intact nuclei were examined. Nuclei lacking hybridization signals were excluded from the evaluation. Results were expressed as a ratio of the numbers of copies of the HER-2/neu gene to the number of the chromosome 17 centromeric markers, with a ratio greater than two being considered amplified. Samples were classified as unamplified when two copies of HER-2/neu and two copies of chromosome 17 were found in the majority of cells and also when the HER-2/neu to chromosome 17 ratio was lower than two.

\subsubsection{Positive}

FISH HER-2/neu/CEP17 ratio 2.2 or greater, or FISH HER-2/neu gene copy number $>6.0$.

\subsubsection{Equivocal}

FISH HER-2/neu/CEP17 ratio 1.8 - 2.2, or FISH HER-2/neu gene copy number 4.0 - 6.0.

\subsubsection{Negative}

FISH HER-2/neu/CEP17 $<1.8$, or FISH HER-2/neu gene copy number $<4.0$. (In conjunction with ASCO \& CAP guidelines). 


\section{Results}

HER-2/neu was evaluated by FISH on forty primary breast cancer FNAs and both by FISH and IHC on the corresponding paraffin sections.

The mean patient age was 42.35 years (range, 22 - 65 years).

All the included cases were diagnosed as infiltrating ductal carcinoma, NOS. Tumor size, grading and staging as well as lymph node status are shown in Table 1.

\subsection{Patients' Criteria}

The mean patient age was 42.35 years (range, 22 - 65 years). The largest group $(21 / 40(52.5 \%))$ had Stage II disease, followed by Stage III (10/40, 25\%), then Stage I $(6 / 40,15 \%)$

Three $(7.5 \%)$ of the patients had known distant metastases at the time of the surgery, and 27 cases $(67.5 \%)$ were negative for lymph node metastases.

\subsection{HER-2/neu FISH on FNAs}

Out of the 40 FNAs, 11 (27.5\%) were amplified, while 29 $(72.5 \%)$ were unamplified.

In unamplified cases, two red signals and two green signals were frequently identified (Figure 1).

Amplified tumors, on the other hand, showed increased number of the red signals either singly or in clusters (Figure 2).

As regards the feasibility of FISH on cytological smears, hybridization was successful in all cases.

Table1. Clinico-pathologic characteristics of the forty breast cancer cases.

\begin{tabular}{ccc}
\hline $\begin{array}{c}\text { Clinico-pathologic } \\
\text { parameter }\end{array}$ & Number of the cases & Percentage \\
\hline Tumor size & 6 & 15 \\
$<2 \mathrm{~cm}$ & 16 & 40 \\
$2-5 \mathrm{~cm}$ & 18 & 45 \\
$>5 \mathrm{~cm}$ & & \\
Tumor grading & 4 & 10 \\
Well differentiated & 30 & 75 \\
Moderately differentiated & 6 & 15 \\
Poorly differentiated & & \\
Lymph nodes status & 27 & 67.5 \\
Negative & 13 & 32.5 \\
Positive & & \\
& 3 & 7.5 \\
Distant metastasis & 37 & 92.7 \\
Absent & & \\
Present & 6 & 15 \\
Tumor staging & 21 & 25 \\
I & 10 & \\
II & & \\
III & & \\
IV & & \\
\hline
\end{tabular}

\subsection{HER-2/neu FISH on FFPE Sections}

Out of the 40 tumors examined, 9 cases $(22.5 \%)$ were amplified (Figure 3), whereas 29 cases $(72.5 \%)$ showed no signals amplification (Figure 4). Two cases were nonevaluable due to lack of signals. Amplification signals were more often distributed in clusters.

\subsection{HER-2/neu Immunohistochemistry}

On IHC, out of the same previous 40 tumors $24(60 \%)$ were negative for the protein expression, while 16 cases (40\%) showed overexpression: ten cases $(62.5 \%)$ were scored as (3+) (Figure 5) and six cases (37.5\%) as (2+) (Figure 6).

\subsection{FISH on FNAs versus FISH on FFPE Sections}

Apart from the two cases that showed lack of signals on

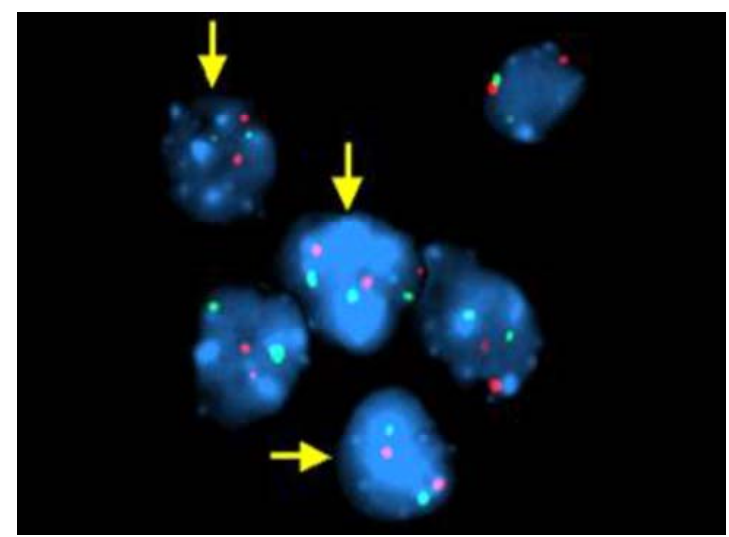

Figure 1. FISH image of a non-amplified Her 2-neu FNA breast cancer case showing normal pattern of 2 green and 2 red signals after hybridization with HER 2-neu probe. (Her2-neu red signals, centromeric green signals).

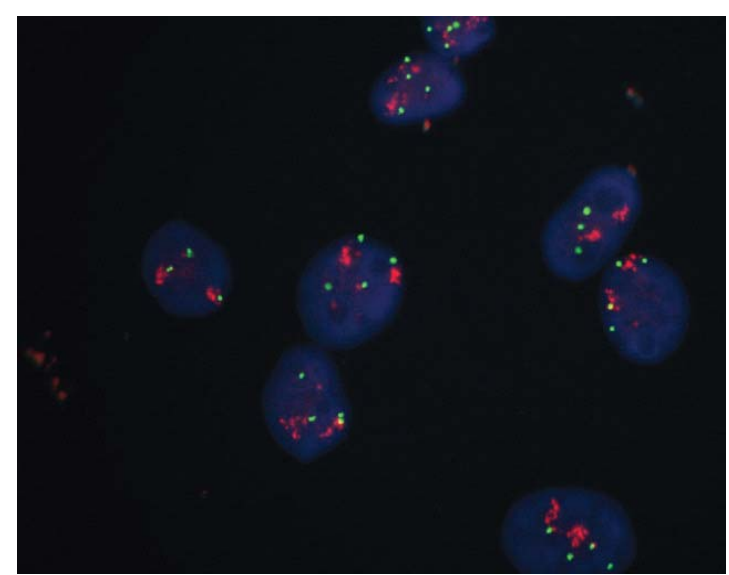

Figure 2. FISH image of an amplified Her 2-neu FNA breast cancer case showing increased number of red signals $\{$ HER2/neu\} than green signals $\{$ Cen 17$\}$. 


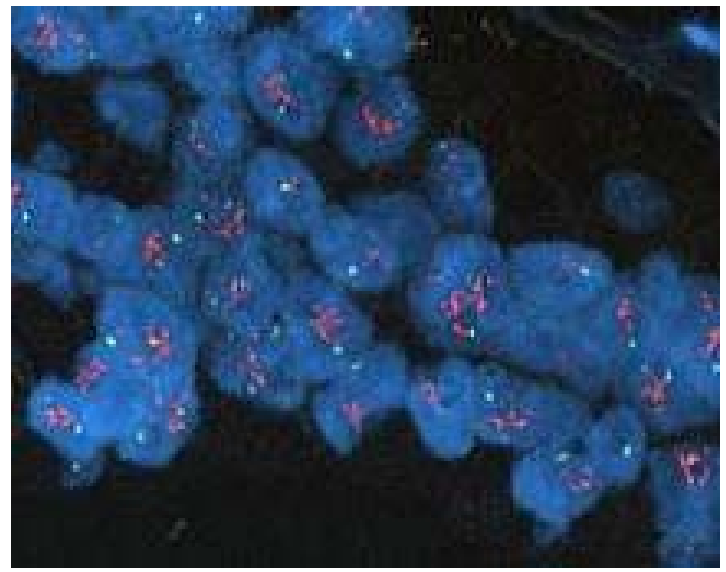

Figure 3. FISH image of the corresponding FFPE section of the FNA shown in Figure 2 with amplified HER-2/neu signals representing cluster pattern. Each tumour cell nucleus demonstrates HER-2/neu signals/CEP 17 signals ratio of $>2.2$.

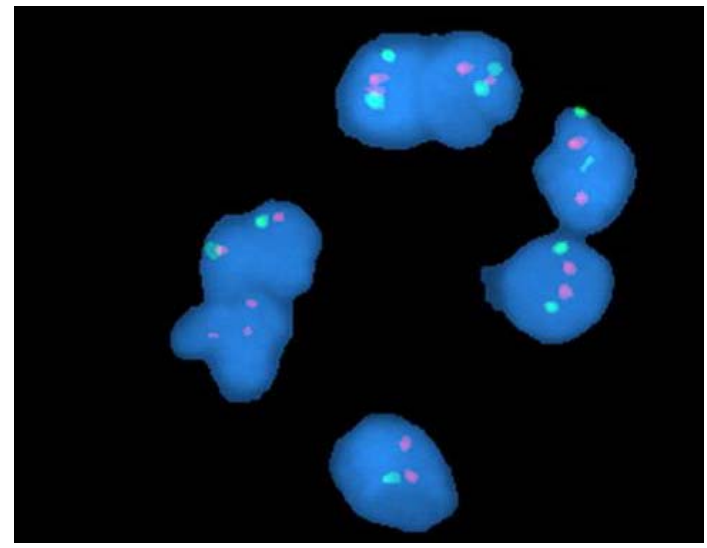

Figure 4. FISH image of the corresponding FFPE section of the FNA shown in Figure 1 showing non-amplified HER2/neu signals. Normal pattern is obtained in most of the scored cells ( 2 green \{cen 17$\}$ and 2 red $\{$ HER-2/neu\} gene signals).

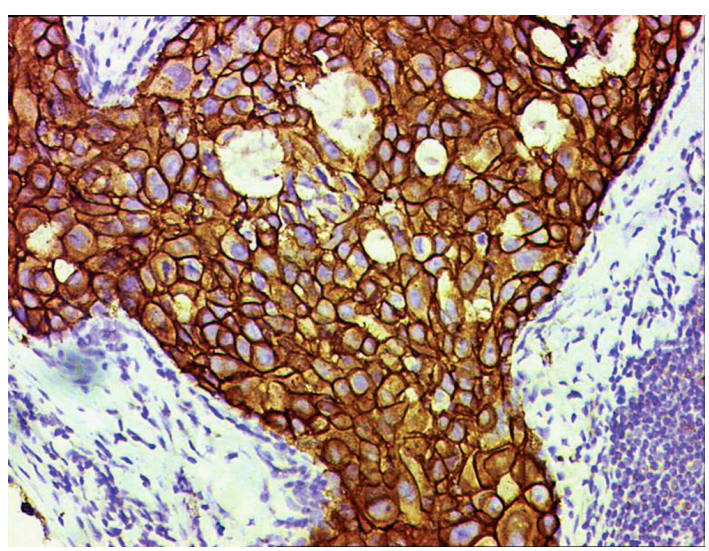

Figure 5. Positive immunostaining for HER-2/neu (score 3+). Uniform intense membrane staining in most invasive tumor cells is seen (Immunoperoxidase, $\times \mathbf{4 0 0}$ ).

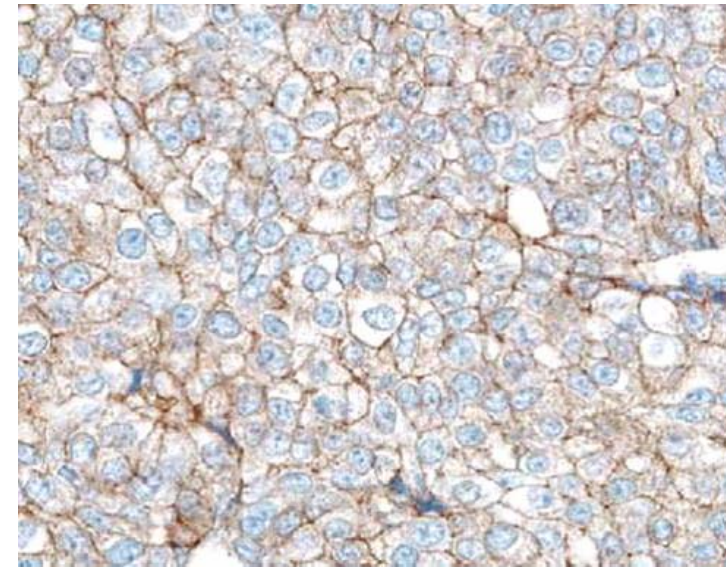

Figure 6. FFPE section showing score 2+ immunostaining for HER-2/neu, complete but weak membrane staining in more than $50 \%$ of tumor cells (Immunoperoxidase, $\times \mathbf{4 0 0}$ ).

FISH histology, all tumors evaluated by FISH on FNAs and on corresponding paraffin-embedded sections showed matched results.

The Measurement of Agreement (Concordance rate) between FISH on FNAs and FISH on FFPE sections was $86.7 \%$ as shown in Table 2.

\subsection{FISH on FNAs versus IHC}

All negative cases (score 0 and $1+$ ) by IHC showed lack of amplification by FISH on FNAs. All $(3+)$ cases by IHC were amplified by FISH on FNAs. As for the 6 cases scored as $(2+)$ by IHC, only one of them showed amplification by FISH on FNA and the other 5 cases were unamplified. The Measurement of Agreement (Concordance rate) between FISH on FNAs and IHC was $72.5 \%$ as shown in Table 2.

\subsection{FISH on FFPE Sections versus IHC}

All negative cases (score 0 and $1+$ ) by IHC showed lack of amplification by FISH on FFPE sections. Eight of the (3+) cases by IHC were amplified by FISH on FFPE sec

Table 2. HER-2/neu results comparing FISH on cytological samples with FISH and IHC on the corresponding histological sections.

\begin{tabular}{ccccc}
\hline FISH on FNA & \multicolumn{5}{c}{ FFPE sections } \\
& \multicolumn{2}{c}{ FISH } & IHC \\
& Amplified & Unamplified Overexpressed & $\begin{array}{c}\text { Not } \\
\text { expressed }\end{array}$ \\
Amplified & 9 & 2 & 11 & 0 \\
Unamplified & 0 & 29 & 5 & 24 \\
$\begin{array}{c}\text { Measurement } \\
\text { of agreement }\end{array}$ & & $86.7 \%$ & & $72.5 \%$ \\
\hline
\end{tabular}


tions. However, 2 of the $(3+)$ cases by IHC were nonevaluable by FISH on FFPE sections. As for the 6 cases scored as $(2+)$ by IHC, only one of them showed amplification by FISH on FFPE sections and the other 5 cases were unamplified. The Measurement of Agreement (Concordance rate) between FISH on FFPE sections and IHC was $89 \%$.

\section{Discussion}

The accuracy of diagnostic assays for HER-2/neu in breast cancer is extremely important as HER-2/neu status is not only a prognostic marker but also predictive of response to chemotherapy, particularly to HER-2/neutargeted therapy such as trastuzumab [24]. HER-2/neu data is also used to predict who will most likely benefit from doxorubicin, taxanes, tamoxifen and other reagents [25]. The diagnostic tests most widely used are IHC and FISH, measuring protein over expression and gene amplification, respectively [24].

Furthermore preoperative determination of the HER$2 /$ neu status is becoming increasingly important to plan for the type of surgery as well as for neo adjuvant chemotherapy in selected patients.

FNAC is widely practiced in Egypt for the preoperative diagnosis of breast cancer and the preoperative detection of lymph node metastasis. Clinicians in our country continue to rely widely on FNAC and consider it an invaluable diagnostic tool. Core needle biopsy is done only in certain conditions [17]. Unfortunately, however the determination of HER2/neu protein expression on cytological preparations was found to be unreliable for clinical use [18]. We therefore opted for the assessment of HER2/neu status in fine needle aspirates using FISH, which was not tried before in our laboratory. By reviewing the literature, the feasibility of HER2/neu amplification detection by FISH in breast cancer FNAs was evaluated by few studies [18-23].

Our study proves the feasibility of HER2/neu amplification detection by FISH on FNA smears as hybridization was successful in all cases. From the technical point of view, FISH signals in cytological smears were more easily visualized than those on FFPE sections. The presence of isolated cells in smears made the signals identification and scoring easier than in tissue sections. This fact was previously reported by Klijanienko et al. [23] and by Bozetti et al. [19] who recommended the use of image analysis in evaluating histological samples. Furthermore, in our work FNA samples preparation for FISH didn't require pretreatment or enzyme digestion with the advantage of less expenses and shorter protocol time than their corresponding FFPE sections. As regards agreement of FISH on FNA smears of breast cancer cases with FISH on corresponding FFPE sections, apart from two cases that showed lack of signals on FISH histology, all tumors evaluated by FISH on FNAs and on corresponding paraffin-embedded sections showed matched results. A good concordance rate of $86.7 \%$ was obtained in our study. If the 2 non-evaluable cases in FFPE sections were omitted from the calculations, then the concordance would be $100 \%$. This is in agreement with Bozetti et al. [19] who found a concordance rate of $91 \%$ between FISH cytology and FISH histology and with Klijanienko et al. [23] who obtained a strong correlation $(\mathrm{P}<0.001)$ between FISH on cytological and histological materials.

Furthermore, in our study, all negative cases (score 0 and $1+$ ) by IHC showed lack of amplification by FISH on FNAs. All 3+ cases by IHC were amplified by FISH on FNAs. Similar results were obtained by McManus DT et al. [21] who detected high amplification by FISH on FNAs from 3 out of the 15 tumors they examined with matched diffuse membranous staining by IHC of the same tumors.

However, a concordance rate of only $72.5 \%$ was found between FISH on FNA smears and IHC in our study. This is attributed to the 6 cases that were given a score of (2+) by IHC and which gave variable results by FISH, as only one of them showed amplification by FISH on FNA and the other 5 cases were unamplified. In the present study, a $100 \%$ agreement was found between the $(3+)$ cases by IHC and their corresponding FISH on FFPE sections except for 2 cases showing complete lack of signals. Similarly a $100 \%$ agreement was found between the score (0) and (1+) cases by IHC and their corresponding FISH on FFPE sections. However, the overall concordance rate between FISH on FFPE sections and IHC was $89 \%$, owing to the variable agreement of the score $(2+)$ cases with their FISH results.

This variable agreement of $2+$ cases with gene amplification was previously encountered in several studies [18,26-28] emphasizing the importance of reevaluation of the HER-2/neu status by FISH in all (2+) cases.

Although several studies evaluating FISH and IHC support the view that FISH has a higher accuracy than IHC [29-31] and that FISH might be a better predictor of response to trastuzumab therapy $[32,33]$, we still use IHC in our laboratory as the first line to triage cases and then the score (2+) ones are further evaluated by FISH. This is because of the lower cost of IHC which is covered by the medical insurance of the patient. Furthermore, IHC is easier to perform and the FISH equipment is not available in our routine pathology laboratory. Therefore we tried to validate the FDA-approved HercepTest that is newly implemented in our laboratory. After modification of some technical issues and interpretation criteria we compared the HercepTest results with the FISH results of the same cases. Only $90 \%$ concordance was reached before start- 
ing this research and $89 \%$ with the research cases. The $\mathrm{ASCO} / \mathrm{CAP}$ guidelines, however, recommend a minimum of $95 \%$ concordance for validation, which we couldn't reach due to the variable agreement of score $(2+)$ cases by IHC. In fact HercepTest has been demonstrated in several studies to produce significant numbers of false positives [31,34-36].

In conclusion, the high concordance rate in the present study between FISH evaluation of HER2/neu gene amplification on FNAC samples and their corresponding FFPE samples indicate that FISH may be a reliable technique for HER2/neu assessment on FNA samples. This is of great significance in our institution where preoperative diagnostic FNAC is widely practiced. Evaluation of HER2/neu gene amplification on FNAC specimen may play a role in planning neoadjuvant chemotherapy in candidate patients, in the management of patients with advanced or recurrent disease and in deciding the type of surgery whether conservative or radical in selected patients. Furthermore, FISH on FNAs gave us better hybridization signals than their corresponding FFPE tissue sections. Finally, we also conclude that all score $(2+)$ cases by IHC should be reevaluated by FISH which is crucial for the patient management. And if our hospital could afford it, we would recommend the use of FISH as the primary method for assessment of all FNAs and FFPE sections of breast cancers to determine the HER-2/neu status.

\section{REFERENCES}

[1] Middle East Cancer Consortium (MECC) Egypt, "The Gharbiah Population-Based Cancer Registry," Ministry of Health and Populations Egypt and Middle East Cancer Consortium, Cairo, 1999.

[2] National Cancer Institue, "Cairo University, Egypt. Cancer Registry 2002-2003,” National Cancer Institue, Cairo.

[3] J. Hannemann, P. Kristel, H. van Tinteren, M. Bontenbal, Q. G. van Hoesel, W. M. Smit, et al., "Molecular Subtypes of Breast Cancer and Amplification of Topoisomerase II Alpha: Predictive Role in Dose Intensive Adjuvant Chemotherapy," British Journal of Cancer, Vol. 95, No. 10, 2006, pp. 1334-41.

[4] R. B. Dickson, R. G. Pestell and M. E. Lippman, "Cancer of the Breast," In: V. Devita, S. Hellman and S. A. Rosenberg, Cancer Principles \& Practice of Oncology, 7th Edition, Lippincott, Williams \& Wilkins, Philadelphia, 2005, pp. 1595-1654.

[5] R. A. Walker, J. M. Bartlett, M. Dowsett, I. O. Ellis, A. M. Hanby, B. Jasani, et al., "HER2 Testing in the UK: Further Update to Recommendations," Journal of Clinical Pathology, Vol. 61, No. 7, 2008, pp. 818-824. doi:10.1136/jcp.2007.054866

[6] D. Varshney, Y. Y. Zhou, S. A. Geller and R. Alsabeh, "Determination of HER-2 Status and Chromosome 17
Polysomy in Breast Carcinomas Comparing HercepTest and PathVysion FISH Assay," American Journal of Clinical Pathology, Vol. 121, No. 1, 2004, pp. 70-77. doi:10.1309/FUQH92B039025LHG

[7] H. J. Burstein, L. N. Harris, R. Gelman, S. C. Lester, R. A. Nunes, C. M. Kaelin, et al., "Preoperative Therapy with Trastuzumab and Paclitaxel Followed by Sequential Adjuvant Doxorubicin/Cyclophosphamide for HER2 Overexpressing Stage II or III Breast Cancer: A Pilot Study," Journal of Clinical Oncology, Vol. 21, No. 1, 2003, pp. 46-53.

[8] M. F. Press, R. S. Finn, D. Cameron, A. Di Leo, C. E. Geyer, I. E. Villalobos, et al., "HER-2 Gene Amplification, HER-2 and Epidermal Growth Factor Receptor mRNA and Protein Expression, and Lapatinib Efficacy in Women with Metastatic Breast Cancer," Clinical Cancer Research, Vol. 14, No. 23, 2008, pp. 7861-7870.

[9] P. L. Fitzgibbons, D. A. Murphy, D. M. Dorfman, P. C. Roche and R. R. Tubbs, "Interlaboratory Comparison of Immunohistochemical Testing for HER2: Results of the 2004 and 2005 College of American Pathologists HER2 Immunohistochemistry Tissue Microarray Survey," Archives of Pathology \& Laboratory Medicine, Vol. 130, No. 10, 2006, pp. 1440-1445.

[10] A. Rhodes, B. Jasani, E. Anderson, A. R. Dodson and A. J. Balaton, "Evaluation of HER-2/neu Immunohistochemical Assay Sensitivity and Scoring on Formalin-Fixed and Paraffin-Processed Cell Lines and Breast Tumors: A Comparative Study Involving Results from Laboratories in 21 Countries," American Journal of Clinical Pathology, Vol. 11, No. 3, 2002, pp. 408-417.

[11] M. B. Lambros, R. Natrajan and J. S. Reis-Filho, "Chromogenic and Fluorescent in Situ Hybridization in Breast Cancer," Human Pathology, Vol. 38, No. 8, 2007, pp. 1105-1022. doi:10.1016/j.humpath.2007.04.011

[12] M. F. Press, G. Sauter, L. Bernstein, I. E. Villalobos, M. Mirlacher, J. Y. Zhou, et al., "Diagnostic Evaluation of HER-2 as a Molecular Target: An Assessment of Accuracy and Reproducibility of Laboratory Testing in Large, Prospective, Randomized Clinical Trials," Clinical Cancer Research, Vol. 11, No. 18, 2005, pp. 6598-6607.

[13] H. Yaziji, L. C. Goldstein, T. S. Barry, R. Werling, H. Hwang, G. K. Ellis, et al., "HER-2 Testing in Breast Cancer Using Parallel Tissue-Based Methods," JAMA Network, Vol. 291, No. 16, 2004, pp. 1972-1977.

[14] L. Arnould, P. Roger, G. Macgrogan, M. P. Chenard, A. Balaton, S. Beauclair, et al., "Accuracy of HER2 Status Determination on Breast Core-Needle Biopsies (Immunohistochemistry, FISH, CISH and SISH vs FISH)," Modern Pathology, Vol. 25, No. 5, 2012, pp. 675-682.

[15] A. C. Wolff, M. E. Hammond, J. N. Schwartz, K. L. Hagerty, D. C. Allred, R. J. Cote, et al., "American Society of Clinical Oncology/College of American Pathologists Guideline Recommendations for Human Epidermal Growth Factor Receptor 2 Testing in Breast Cancer," Journal of Clinical Oncology, Vol. 25, No. 1, 2007, pp. 118-145. doi:10.1200/JCO.2006.09.2775

[16] I. O. Ellis, J. Bartlett, M. Dowsett, S. Humphreys, B. 
Jasani, K. Miller, et al., "Best Practice No 176: Updated Recommendations for HER2 Testing in the UK," Journal of Clinical Pathology, Vol. 57, No. 3, 2004, pp. 233-237. doi:10.1136/jcp.2003.007724

[17] M. Abdel-Hadi, G. F. Abdel-Hamid, N. Abdel-Razek and R. K. Fawzy, "Should Fine-Needle Aspiration Cytology Be the First Choice Diagnostic Modality for Assessment of All Nonpalpablebreast Lesions? The Experience of a Breast Cancer Screening Center in Alexandria, Egypt," Diagnostic Cytopathology, Vol. 38, No. 12, 2010, pp. 880889. doi: $10.1002 /$ dc. 21305

[18] B. G. Beatty, R. Bryant, W. Wang, T. Ashikaga, P. C. Gibson, G. Leiman, et al., "HER-2/neu Detection in FineNeedle Aspirates of Breast Cancer: Fluorescence in Situ Hybridization and Immunocytochemical Analysis," American Journal of Clinical Pathology, Vol. 122, No. 2, 2004, pp. 246-255.

[19] C. Bozzetti, R. Nizzoli, A. Guazzi, M. Flora, C. Bassano, P. Crafa, et al., "HER-2/neu Amplification Detected by Fluorescence in Situ Hybridization in Fine Needle Aspirates from Primary Breast Cancer," Annals of Oncology, Vol. 13, No. 9, 2002, pp. 1398-1403. doi:10.1093/annonc/mdf217

[20] A. Mezzelani, L. Alasio, C. Bartoli, M. G. Bonora, M. A. Pierotti, F. Rilke, et al., "c-erbB2/neu Gene and Chromosome 17 Analysis in Breast Cancer by FISH on Archival Cytological Fine-Needle Aspirates," British Journal of Cancer, Vol. 80, No. 3-4, 1999, pp. 519-525.

[21] D. T. McManus, A. H. Patterson, P. Maxwell, M. W. Humphreys and N. H. Anderson, "Fluorescence in Situ Hybridisation Detection of erbB2 Amplification in Breast Cancer Fine Needle Aspirates," Molecular Pathology, Vol. 52, No. 2, 1999, pp. 75-77.

[22] G. Sauter, G. Feichter, J. Torhorst, H. Moch, H. Novotna, U. Wagner, et al., "Fluorescence in Situ Hybridization for Detecting erbB-2 Amplification in Breast Tumor Fine Needle Aspiration Biopsies," Acta Cytologica, Vol. 40, No. 2, 1996, pp. 164-173.

[23] J. Klijanienko, J. Couturier, M. Galut, A. K. El-Naggar, Z. Maciorowski, E. Padoy, et al., "Detection and Quantitation by Fluorescence in Situ Hybridization (FISH) and Image Analysis of HER-2/neu Gene Amplification in Breast Cancer Fine-Needle Samples," Cancer, Vol. 87, No. 5, 1999, pp. 312-318.

[24] A. M. Gown, "Current Issues in ER and HER2 Testing by IHC in Breast Cancer," Modern Pathology, Vol. 21, No. S8-S15, 2008, p. 34.

[25] A. Thor, "Applications of Molecular Analysis to Small Biopsy and Cytology Specimens for Diagnosis and Targeted Therapy. HER2/neu (erbB2) Analysis on FNA Smears and Core Biopsies of Primary or Metastatic Breast Cancer," PSC/USCAP, 2008. http://pathologyportal.org/97th/pdf/companion16h03.pdf

[26] M. F. Press, D. J. Slamon, K. J. Flom, J. Park, J. Y. Zhou and L. Bernstein, "Evaluation of HER-2/neu Gene Amplification and Overexpression: Comparison of Frequently Used Assay Methods in a Molecularly Characterized Cohort of Breast Cancer Specimens," Journal of Clinical Oncology, Vol. 20, No. 14, 2002, pp. 3095-3105.
[27] K. I. Goud, S. Dayakar, K. Vijayalaxmi, S. J. Babu and P. V. Reddy, "Evaluation of HER-2/neu Status in Breast Cancer Specimens Using Immunohistochemistry (IHC) \& Fluorescence In-Situ Hybridization (FISH) Assay," Indian Journal of Medical Research, Vol. 135, No. 3, 2012, pp. 312-317.

[28] R. D. Mass, M. F. Press and S. Anderson, "Improved Survival Benefit from Herceptin (Trastuzumab) in Patients Selected by Fluorescence in Situ Hybridization (FISH) (Abstract)," Proc Am Soc Clin Oncol, Vol. 20, No. 22a, 2001.

[29] S. Kakar, N. Puangsuvan, J. M. Stevens, R. Serenas, G. Mangan, S. Sahai, et al., "HER-2/neu Assessment in Breast Cancer by Immunohistochemistry and Fluorescence in Situ Hybridization: Comparison of Results and Correlation with Survival," Journal of Molecular Diagnostics, Vol. 5, No. 3, 2000, pp. 199-207.

[30] M. F. Press, L. Bernstein, P. A. Thomas, L. F. Meisner, J. Y. Zhou, Y. Ma, et al., "HER-2/neu Gene Amplification Characterized by Fluorescence in Situ Hybridization: Poor Prognosis in Node-Negative Breast Carcinomas," Journal of Clinical Oncology, Vol. 15, No. 8, 1997, pp. 2894 2904.

[31] G. Pauletti, S. Dandekar, H. Rong, L. Ramos, H. Peng, R. Seshadri, et al., "Assessment of Methods for TissueBased Detection of the HER-2/neu Alteration in Human Breast Cancer: A Direct Comparison of Fluorescence in Situ Hybridization and Immunohistochemistry," Journal of Clinical Oncology, Vol. 18, No. 21, 2000, pp. 36513664.

[32] C. L. Vogel, M. A. Cobleigh, D. Tripathy, J. C. Gutheil, L. N. Harris, L. Fehrenbacher, et al., "Efficacy and Safety of Trastuzumab as a Single Agent in First-Line Treatment of HER2-Overexpressing Metastatic Breast Cancer," Journal of Clinical Oncology, Vol. 20, No. 3, 2002, pp. 719-726.

[33] M. A. Cobleigh, C. L. Vogel, D. Tripathy, N. J. Robert, S. Scholl, L. Fehrenbacher, et al., "Multinational Study of the Efficacy and Safety of Humanized Anti-HER2 Monoclonal Antibody in Women Who Have HER2-Overexpressing Metastatic Breast Cancer That Has Progressed after Chemotherapy for Metastatic Disease," Journal of Clinical Oncology, Vol. 17, No. 9, 1999, pp. 2639-2648.

[34] T. W. Jacobs, A. M. Gown, H. Yaziji, M. J. Barnes and S. J. Schnitt, "Specificity of HercepTest in determining HER2/neu Status of Breast Cancers Using the United States Food and Drug Administration-Approved Scoring System," Journal of Clinical Oncology, Vol. 17, No. 7, 1999, pp. 1983-1987.

[35] P. C. Roche and J. N. Ingle, "Increased HER2 with US Food and Drug Administration-Approved Antibody," Journal of Clinical Oncology, Vol. 17, No. 1, 1999, p. 434.

[36] A. Lebeau, D. Deimling, C. Kaltz, A. Sendelhofert, A. Iff, B. Luthardt, et al., "Her-2/neu Analysis in Archival Tissue Samples of Human Breast Cancer: Comparison of Immunohistochemistry and Fluorescence in Situ Hybridization," Journal of Clinical Oncology, Vol. 19, No. 2, 2001, pp. 354-363. 